\title{
Reflexiones críticas en torno a la regulación de las cooperativas no agropecuarias en Cuba
}

(Critical reflections on the regulation of non-agricultural cooperatives in Cuba)

\author{
Natacha Teresa Mesa Tejeda ${ }^{1}$ \\ Universidad de la Habana
}

Reclbído: 09.05.2014

Aceptado: 01.09.2014

Sumario: I. Ideas Introductorias. II. Análisis de la regulación jurídica de las Cooperativas no Agropecuarias en Cuba. 2.1. Clasificación. 2.2. Constitución. 2.3. Régimen económico. 2.4. Órganos de la Cooperativa. 2.4.1. Asamblea General 2.4.2. Junta Directiva. 2.4.3. Administrador o Consejo Administrativo. 2.4.4. Comisión de Control y Fiscalización. III. Ideas Conclusivas. IV. Bibliografía.

Resumen: Las cooperativas no agropecuarias, a partir de diciembre del 2012, constituyen un nuevo sujeto integrante del sistema empresarial cubano. Su constitución puede contribuir al desarrollo económico del país, no obstante se requieren de diversos factores que hagan posible su acertada implementación, dentro de los que ocupa un papel preponderante la necesidad de una legislación que regule de manera general el fenómeno cooperativo en Cuba, pues hoy existen varias normativas para regular este fenómeno. En el presente artículo se realiza un análisis crítico de algunos aspectos contenidos en la regulación jurídica de las cooperativas no agropecuarias en Cuba, como son: naturaleza jurídica, proceso de constitución, régimen económico y órganos,- con el objetivo de contribuir a su perfeccionamiento.

Palabras clave: cooperativas, constitución, régimen económico, organización.

Abstract: Since December 2012, non-farming cooperatives constitute a new subject integrating the Cuban economic system. However its development can contribute to the economic development of our country, it does require the existence of some factors to make possible its right implementation; among them we can point the necessity of the existence of a general legislation regulating this figure in Cuba, since today we have a variety of regulations around it. The present article contains a critical analysis of some of the aspects

1 Profesora Auxiliar Derecho Mercantil, Facultad de Derecho, Universidad de la Habana, correo electrónico: natasha@lex.uh.cu 
of the non-farming cooperatives regulation in Cuba -its juridical nature, its constitution process, its economic regime, and its internal administration- all of these with ultimate goal of improving its general regulation.

Key words: cooperative, constitution, economic regime, organization. 


\section{Ideas introductorias}

Si bien la empresa estatal, actualmente, continúa desempeñando un papel preponderante en el sistema empresarial cubano, no podemos afirmar que sea el único eslabón que lo integra. Con la aprobación de los Lineamientos de la Política Económica y Social del Partido (en lo adelante Lineamientos) que ha propiciado la actualización del modelo económico cubano se pretende, entre otros aspectos, garantizar el desarrollo económico del país. Para ello se promueve la utilización de otras formas de gestión no estatal en nuestra economía. Una de estas formas de gestión no estatal es la Cooperativa, pero esta vez, su ámbito de desarrollo se extiende a otros sectores de la economía cubana. Así se encuentra reflejados en los Lineamientos 2 y 25 al 29.

La cooperativa no es una figura de reciente aparición en nuestro país, encontramos su presencia en el sector agrícola desde hace aproximadamente tres décadas, de ahí que tradicionalmente el único tipo de cooperativa que se constituyó en Cuba fue la cooperativa agropecuaria.

En consecuencia, en diciembre de 2012 se promulga el paquete legal contentivo de la normativa jurídica que regularía lo relativo a las cooperativas no agropecuarias, integrado por las siguientes normas jurídicas: Decreto Ley 305 «De las Cooperativas no Agropecuarias (CNA)»; DecretoLey 306 «Del régimen especial de Seguridad Social de los socios de las CNA», Decreto 309 «Reglamento de las cooperativas no agropecuarias de primer grado», Resolución 570/12 del Ministro de Economía y Planificación sobre el Proceso de Licitación, Resolución 427/2012 de la Ministra de Finanzas y Precios sobre el Régimen Tributario. ${ }^{2}$

En nuestro contexto la cooperativa ofrece varias ventajas, entre las que podemos destacar: surgimiento de un nuevo sujeto de gestión, se crean nuevos puestos de trabajo, aumenta la competitividad en el sector empresarial toda vez que el Estado ya no es el único oferente, la inexistencia de un salario mínimo permite incentivar al trabajador los que, conscientes de los beneficios que ofrece la figura, podrán aumentar la eficiencia y la calidad en la producción o el servicio que prestan. Aquellas cooperativas dedicadas a la producción permitirán, además, sustituir importaciones.

En el presente artículo se realiza un análisis crítico de algunos aspectos contenidos en la regulación jurídica de las cooperativas no agropecuarias en Cuba, como son: naturaleza jurídica, proceso de constitución, régimen económico y órganos, por la importancia que los mismos

2 Publicados en Gaceta Oficial Extraordinaria No. 053 de 11 de diciembre de 2012. 
poseen. La esencia del análisis es legislativo precisamente porque al momento de la redacción de este artículo, aún no ha transcurrido un año de que se constituyeran las primeras cooperativas en el sector no agropecuario $^{3}$ (junio de 2013) y por tanto, las experiencias acumuladas hasta la fecha en torno al funcionamiento de las mismas no son del todo suficientes. De esta manera pretendemos, contribuir al perfeccionamiento de la legislación y comenzar a sentar las bases de lo que pudiera ser un Derecho Cooperativo cubano.

Estamos en presencia de un artículo que se caracteriza por ser reflexivo y que tributa al proyecto de investigación que posee el Departamento de Asesoría Jurídica e Internacional de la Facultad de Derecho de la Universidad de la Habana, titulado "Las cooperativas. Una alternativa viable para el desarrollo económico en Cuba,».

\section{Análisis de la regulación jurídica de las Cooperativas no Agropecuarias en Cuba}

Al analizar la regulación jurídica de las cooperativas no agropecuarias, y realizar valoraciones de los aciertos y desaciertos del legislador cubano en esta materia tendríamos contenido para realizar diversos artículos. Uno de los elementos que podríamos tomar como punto de partida sería la ausencia de basamento constitucional de la regulación jurídica de la cooperativa. No obstante, preferimos circunscribir nuestro análisis, a cuestiones como la naturaleza jurídica, el proceso de constitución, el régimen económico y los órganos de la cooperativa, los que se encuentran regulado en el Decreto Ley 305 y Decreto 309.

Lo primero que esta autora considera importante analizar es lo relativo a la naturaleza jurídica. La determinación de la naturaleza jurídica para nada constituye un tema formal, su importancia transita por aspectos tan sensibles como la determinación del régimen jurídico aplicable y por tanto en su ubicación como sujeto dentro de una rama del Derecho. Es válido destacar que, el tema de la naturaleza jurídica de las cooperativas no ha sido para nada pacífico en la doctrina, en torno a este particular existen varias tesis que intentan explicarla. Autores como Gadea, Sacristán y Vargas Vasserot la han agrupado en tres posiciones básicas: las que la consideran una asociación; una sociedad y por último, las que la consideran una categoría autónoma. Las legislaciones

3 Hasta la fecha de redacción del presente artículo se encontraban constituidas un número de 224 cooperativas de un total de 498 autorizadas, estos datos han sido extraídos del Periódico Granma, en su edición de fecha de 3 de Marzo de 2014. 
cooperativas internacionales de igual manera dan fe de esta falta de consenso que existe en torno a este particular. ${ }^{4}$

El comentario anterior nos conduce a realizarnos la interrogante siguiente, ¿Qué posición ha asumido el legislador cubano? En el artículo 2.1 del mencionado Decreto Ley 305 se define a la cooperativa, como «() organización con fines económicos y sociales, que se constituye voluntariamente sobre la base del aporte de bienes y derechos y se sustenta en el trabajo de sus socios, cuyo objetivo general es la producción de bienes y la prestación de servicios mediante la gestión colectiva, para la satisfacción del interés social y el de los socios.

La cooperativa tiene personalidad jurídica y patrimonio propio; usa, disfruta y dispone de los bienes de su propiedad, cubre sus gatos con sus ingresos y responde de sus obligaciones con su patrimonio.»

Esta definición responde en líneas generales a las características tradicionalmente aceptadas para las cooperativas a través de la definición ofrecida por la Alianza Cooperativa Internacional (ACI) en $1995^{5}$. Sin embargo, de la lectura del precepto colegimos que el legislador cubano utiliza una fórmula muy genérica al calificarla como organización, por lo que no la reconoce expresamente ni como una sociedad ni como una asociación. Sin embargo, otros artículos del texto legal nos conducen a pensar en la posibilidad de concebir a la cooperativa como una sociedad ya que se utiliza el término de $\operatorname{socios}^{6}$ y no el de miembros o asociados, en el artículo 4 apartado f) se destaca la carencia del ánimo especulativo en la cooperativa lo que nos pudiera llevar a comprender a la cooperativa como una asociación. ${ }^{7}$

Sin embargo existe otra legislación que pudiera entrar en contradicción con la concepción asumida por el legislador cubano en el Decreto Ley 305, se trata del Código de Comercio. Esta normativa en su artículo 124 establece que: "Las Compañías mutuas de seguros contra incendios,

4 GADEA, E.; SACRISTÁN, F. y VARGAS VASSEROT, C.: Régimen jurídico de la sociedad cooperativa del siglo Xx I. Realidad actual y propuestas de reforma, Ed. Dykison, Madrid, 2009.

5 Declaración de Manchester sobre la Identidad Cooperativa, de 23 de septiembre de 1995.

6 Vid. artículos 2.1, 4, 10.1, 11 D/L 305

7 Artículo 4.- - "Las cooperativas se sustentan en los principios siguientes: f) Responsabilidad social, contribución al desarrollo planificado de la economía y al bienestar de sus socios y familiares: Los planes de las cooperativas tienen como objetivo contribuir al desarrollo económico y social sostenible de la nación, proteger el medio ambiente, desarrollar sus actividades sin ánimo especulativo y garantizar el cumplimiento disciplinado de las obligaciones fiscales y otras. Trabajan por fomentar la cultura cooperativista y por las satisfacciones de las necesidades materia-les, de capacitación, sociales, culturales, morales y espirituales de sus socios y familiares. 
de combinaciones tontinas sobre la vida para auxilios a la vejez y de cualquiera otra clase y las cooperativas de producción, de crédito o de consumo, sólo se considerarán mercantiles y quedarán sujetas a las disposiciones de este Código, cuando se dedicaren a actos de comercio extraños a la mutualidad o se convirtieren en Sociedades a prima fija.»

Es evidente que, conforme dispone el Código de Comercio las cooperativas, de producción, consumo o créditos que realicen actos ajenos o extraños a la mutualidad se considerarán mercantiles. Con respecto al carácter mutualista ${ }^{8}$ podemos afirmar que no es un elemento distintivo de las cooperativas no agropecuarias cubanas, toda vez que, sus socios además de satisfacer sus necesidades pueden realizar ventas directas sin intermediarios, una vez que hayan cumplido sus compromisos con el Estado, en los casos que corresponda. ${ }^{9}$ Por lo que, los únicos destinatarios o beneficiarios de los productos o servicios de las cooperativas no agropecuarias no solo son los cooperativistas, sino también el público en general, de modo que, se difumina en estas cooperativas el carácter mutualista. En consecuencia, lo antes expuesto podría generar que, de conformidad con lo que establece el Código de Comercio, las cooperativas sean consideradas un tipo de sociedad mercantil especial dentro de las reguladas en él, y por tanto se le aplique en lo pertinente sus disposiciones. Es opinión de la autora que este es un tema que bien merece ser objeto de un artículo posterior, por ahora solo colocamos la semilla.

\section{II.1. Clasificación}

El legislador cubano establece una clasificación de las cooperativas, teniendo en cuenta la naturaleza de sus socios o lo que es lo mismo, la forma de organizarse. Para ello dispone que las cooperativas podrán ser de dos tipos: de primer grado y de segundo grado. Las de primer grado se integran mediante la asociación voluntaria de al menos tres personas naturales. ${ }^{10} \mathrm{Un}$ elemento interesante que resalta de la lectura

8 Amorín, M.; Algorta, P.: Sociedades Cooperativas Sistema y Derecho Cooperativo, Ed. La Ley Montevideo, pagina 42, 2010, (versión de pre impresión facilitada por los autores en medio magnético para la presente investigación)

9 Vid. Artículo 66. 1 Decreto 309/12.

10 Artículo 5.1. - «Las cooperativas pueden ser de primer o de segundo grado.

2. Es de primer grado la cooperativa que se integra mediante la asociación voluntaria de al menos tres personas naturales.

3. Es de segundo grado la cooperativa que se integra por dos o más cooperativas de primer grado con el objetivo de organizar actividades complementarias afines o que agreguen valor a los productos y servicios de sus socios, o de realizar compras y ventas conjuntas, con vistas a lograr mayor eficiencia.» 
del precepto mencionado es que a diferencia del tratamiento que en diversas legislaciones se le ofrece a este particular solo pueden integrar las cooperativas no agropecuarias de primer grado en Cuba, las personas naturales, las que, necesariamente deben participar con su trabajo en la actividad que constituye el objeto social de la cooperativa. No se establece para su constitución límite máximo de socios, consideramos que esto responde a la acogida por el legislador cubano de uno de los principios del cooperativismo internacional: puertas abiertas conforme al mismo los socios pueden incorporarse libremente a la cooperativa así como causar baja de ella.

Las referidas cooperativas pueden constituirse bien de manera voluntaria o por iniciativa estatal, para lo cual la ley le confiere tres posibilidades: a) a partir del patrimonio integrado por los aportes dinerarios de los socios, b) por personas naturales que decidan asociarse entre sí con la finalidad de adquirir conjuntamente insumos y servicios, comercializar productos y servicios, o emprender otras actividades económicas, conservando los socios la propiedad sobre sus bienes; c) a partir de medios de producción del patrimonio estatal, tales como inmuebles y otros, que se decida gestionar de forma cooperativa y para ello puedan cederse estos bienes, por medio de arrendamiento, usufructo $u$ otras formas legales que no impliquen la transmisión de la propiedad. ${ }^{11}$ Es criterio de esta autora que las cooperativas constituidas por iniciativa estatal merecen especial atención, toda vez que no nace de los socios, a partir de sus necesidades, la iniciativa de unirse y crear una cooperativa. Se trata de entidades o empresas estatales que se decide administrativamente gestionar bajo la forma cooperativa. Esto puede ser peligroso pues pueden cometerse errores en este proceso que den al traste con que se desvirtúe la esencia de la figura. Consideramos que, en este proceso de tránsito hacia la forma de gestión no estatal de referencia, deben tenerse en cuenta varios elementos y uno de ellos es el de la educación e información, es decir que los antiguos trabajadores de la empresa, hoy socios de la cooperativa, conozcan las características y beneficios que ofrece la misma. La necesidad de que los cooperativistas estén debidamente informados y educados bajo una concepción cooperativa obedece, entre otros aspectos, al hecho que, tomando como base el principio cooperativo de la autogestión, necesitan entonces estar informados, capacitados para conducirla de manera certera en función de alcanzar el objeto social propuesto.

Las cooperativas de segundo grado se integran por dos o más cooperativas de primer grado con el objetivo de organizar actividades com-

11 Vid. Artículo 5.1 y 5.2 Decreto Ley 305/12. 
plementarias afines o que agreguen valor a los productos y servicios de sus socios, o de realizar compras y ventas conjuntas, con vistas a lograr mayor eficiencia. ${ }^{12}$ En las páginas siguientes dedicaremos el análisis a las cooperativas de primer grado, en tanto la normativa que regula las de segundo grado a la fecha de la redacción del presente artículo no se ha publicado.

A diferencia de las tendencias contenidas en las legislaciones cooperativas internacionales, en el Decreto 309, no se establece ninguna clasificación en función del objeto o actividad a la que se dedique la cooperativa, de modo que podría entenderse que podrá dedicarse a cualquier actividad, como se desprende de la amplia formulación contenida en la definición cuando señala como objetivo general de la cooperativa «la producción de bienes y la prestación de servicios». Lo cierto es que no se trata de cualquier tipo de servicio, como podremos ver más adelante las cooperativas necesitan de autorización para constituirse, recayendo dicha autorización sobre varios aspectos y uno de ellos es el objeto social, por lo que, corresponde al ente que autoriza la creación de las cooperativas pronunciarse en torno a la factibilidad o no del objeto social propuesto por los futuros cooperativistas. Hasta la fecha en que se redacta el presente artículo, algunos de los principales sectores en los que se han constituido cooperativas no agropecuarias en la esfera de los servicios son: comercio, gastronomía, construcción, transporte, servicios contables.

\section{II.2 Proceso de constitución}

Como toda persona jurídica, la cooperativa, necesita cumplir determinadas exigencias legales para su constitución, las que no deben ser consideradas como meras formalidades sino que constituyen requisitos sine qua non para la creación de las mismas. Estos son: Autorización Gubernamental, Escritura Pública y Estatutos formalizados ante Notario, así como la inscripción en el Registro Mercantil.

La autora considera que el proceso de constitución se caracteriza por ser muy centralizado y dilatado, lo cual puede constituir un elemento poco favorable y que desestimule la constitución y desarrollo de las cooperativas en Cuba.

Durante el iter constitutivo la solicitud transita por varios niveles, desde el nivel municipal, provincial así como por las entidades rectoras de la actividad, la Comisión Permanente para la Implementación y De-

12 Vid. Artículo 5.3 Decreto Ley 305/12. 
sarrollo y termina en el Consejo de Ministros, órgano que deberá, escuchado el parecer de los que lo antecedieron, decidir si se aprueba o no la cooperativa. En este sentido consideramos que, es excesiva la intervención administrativa y que, por tanto, este proceso debe flexibilizarse y otorgarle realmente autonomía a los gobiernos locales para que decidan sobre la procedencia o no de la cooperativa. Dado el caso que no se considere procedente descentralizar el poder de decisión en esta materia, bien pudiera pensarse la posibilidad de crear un órgano especializado en cooperativismo que tenga a su cargo el fomento, impulso, desarrollo y control de las cooperativas en nuestro país.

Otro elemento negativo que consideramos oportuno destacar es la ausencia, en la legislación, del término para que la administración ofrezca una respuesta, lo que sin lugar a dudas, puede constituir otro elemento desestimulante sobre todo para las cooperativas no agropecuarias que se constituyen por la voluntad de sus miembros.

Una vez conferida la autorización los futuros socios disponen de 60 días hábiles para acudir ante Notario con el proyecto de estatutos e iniciar el proceso de constitución que culmina con la inscripción en el Registro Mercantil.

A grandes rasgos, han quedado expuestas algunas de las características que distinguen al proceso constitutivo de las cooperativas no agropecuarias en Cuba, las que se resumen en: ser un proceso dilatado, que tiene lugar a través de una secuencia de actos que tienen como objetivo final lograr la inscripción registral y con ello la constitución de la cooperativa, caracterizado por una fuerte intervención administrativa desde que se presenta la solicitud hasta que se inscribe en el Registro.

\section{II.3. Régimen Económico}

Lo relativo al régimen económico de la cooperativa se encuentra regulado en los artículos del 20 al 25 del Decreto Ley 305 así como del 46 al 51 del Decreto 309. Debido a lo poco coherente que resultan algunos de sus artículos, son varios los comentarios que pueden ofrecerse a la regulación de este particular.

En primer lugar es de destacar que, a nuestro juicio, no existe una concepción clara del legislador, de la diferencia existente entre capital y patrimonio, lo cual se evidencia del análisis de la Sección Primera del capítulo V del Decreto 309, la que se denomina «Del Patrimonio» y sin embargo en su articulado regula, fundamentalmente, lo relativo al capital de la cooperativa. Considera esta autora que la sección debió lla- 
marse del Capital y Patrimonio de la cooperativa, toda vez que su contenido regula fundamentalmente lo referido a ambas categorías. En igual sentido, el artículo $22^{13}$ constituye otra evidencia de lo planteado anteriormente, una interpretación del precepto nos permitiría comprender que se está haciendo referencia en lugar del patrimonio, al capital de la cooperativa, ya que al decir de Sánchez Calero ${ }^{14}$, el patrimonio incluye no solo el capital, derechos y bienes, sino también las obligaciones y deudas de la cooperativa, es decir incluye tanto el activo como el pasivo. El capital, por su parte, se caracteriza por ser variable, lo cual es una consecuencia del principio cooperativo de puertas abiertas cuya función fundamental es la de generar productividad, toda vez que se dedica al desarrollo del objeto social. ${ }^{15}$

Otro de los aspectos a meditar en esta temática es el relativo a qué pueden aportar los socios para constituir una cooperativa de primer grado. De la lectura de la normativa podemos inferir que, para la constitución de la cooperativa y la creación de lo que el legislador ha denominado «capital de trabajo inicial», solo pueden ser objetos de aportación el dinero así como derechos de créditos bancarios, obtenidos con el objetivo de constituir la cooperativa. ${ }^{16}$ A partir de lo expuesto, consideramos que en lo que a aportes respecta, el legislador asumió un criterio muy reduccionista, ya que del tenor literal de los artículos se desprende que solo se considerarán aportes los dinerarios, cerrando las puertas a la posibilidad de efectuar otras aportaciones diferentes al dinero, dígase bienes, otros derechos distintos a los de créditos o por qué no, trabajo. Esta limitación constituye, a nuestro juicio, un factor que pudiera incidir de manera negativa en el desarrollo del cooperativismo en Cuba. Un análisis de algunas legislaciones foráneas, como es el caso de la mexicana y argentina, y otra del continente europeo, es el caso de la española, nos permitió constatar que en todas se le reconoce a los socios la posibilidad de realizar no solo aportaciones de dinero, sino también de bienes, derechos y trabajo. Al decir de Rodríguez Musa, capital y trabajo en las cooperativas no son antagónicos, tal y como su-

13 Artículo 22.- El patrimonio de la cooperativa está integrado por el capital de trabajo inicial y todos los demás bienes y derechos cuya titularidad adquiere la cooperativa de forma lícita.

14 Sánchez Calero, F. y Sánchez Calero, J.:: Instituciones de Derecho Mercantil, Tomo I, Editorial Thomson-Aranzadi, 30 Edición, Navarra, 2006.

15 AMORín, M.; AlgoRTA, P.: Sociedades Cooperativas Sistema y Derecho Cooperativo, Ed. La Ley Montevideo, pagina 45, 2010, (versión de pre impresión facilitada por los autores en medio magnético para la presente investigación).

16 Vid. artículos 6, 21 apartado 1 y 2 del D/L 305/12 y artículos 46 y 48 Decreto $309 / 12$. 
cede en las sociedades capitalistas, el primero es un medio para el fortalecimiento y la rentabilidad. ${ }^{17}$

Por otro lado, tal y como comentábamos en el párrafo anterior, artículos como el 23 del Decreto Ley 305 dan lugar a la duda. En él se establece: "Los socios de la cooperativa, con independencia de cualquier otro aporte que hagan, están en la obligación de participar con su trabajo». De la lectura del precepto se colige que es posible que los socios puedan realizar otras aportaciones distintas al dinero. En tal sentido nos preguntamos, ¿además del dinero y los derechos de créditos, qué más pudiera ser objeto de aportación? ¿En qué momento se pueden realizar las mismas? ¿Cómo se realizaría el proceso de valuación de esos aportes no dinerarios? ¿En qué concepto se realiza la aportación? Dada la ambigüedad del precepto estas interrogantes no pueden ser respondidas. Somos del criterio que la determinación, en una futura normativa, de estas cuestiones resulta transcendental no solo para los socios sino también para la cooperativa.

El capital de trabajo inicial es una partida que integra el patrimonio, puede ser desembolsado íntegramente o de manera aplazada, corresponde a los socios determinar en los estatutos el modo en el que ellos deberán cumplir con su obligación. Los socios, al momento de la formalización ante Notario de la escritura pública, deben justificar la realización de aportaciones dinerarias mediante la presentación de una certificación del Banco que acredite el depósito ${ }^{18}$.

\section{II.4. Órganos de la cooperativa}

Las personas jurídicas, cual ficción del Derecho, necesitan de órganos para crear la voluntad social así como para actuar frente a terceros. En nuestro país, las cooperativas no agropecuarias cuentan con una estructura orgánica, integrada por: Asamblea General, Junta Directiva, Órgano de Administración y Comisión de Control y Fiscalización, los que se encuentran regulados tanto en el Decreto Ley 305 como en el Decreto 309 ambos del 2012.

\section{II.4.1. Asamblea General}

La Asamblea General es el órgano superior de dirección. Se integrará por todos los socios, los cuales poseen voz y voto, lo que cons-

17 RodríGuez, O.: La cooperativa como figura jurídica. Perspectivas constitucionales en Cuba para su aprovechamiento en otros sectores de la economía nacional diferentes al Agropecuario, Dykinson, S.L., página 50, Madrid, 2011.

18 Vid. artículo 48 Decreto 309/12. 
tituye una muestra del principio cooperativo de gestión democrática, según el cual cada socio posee un voto más allá de la participación que posea en la cooperativa. Estará presidida la Asamblea por un Presidente, su sustituto y un secretario, quienes serán miembros de la cooperativa.

Es un órgano asambleario, sus atribuciones se encuentran determinadas en el artículo $37^{19}$ del Decreto 309. En el propio texto legal, en su artículo 38, también se determinan expresamente, las facultades del Presidente de la Asamblea, lo que hace que podamos pensar en su reconocimiento como un órgano unipersonal. A solo dos de ellas quisiéramos referirnos: a) Ejercer la representación de la cooperativa frente a los propios socios y a terceros, b) Suscribir contratos en los que sea parte la cooperativa, precisamente por la importancia que las mismas poseen. La representación de la cooperativa como atribución cuya titularidad pertenece al Presidente de la cooperativa es otro aspecto, que en nuestra consideración, no se encuentra bien regulado. En la mencionada legislación, se presenta sin límites y esto se convierte en un tema sensible si tenemos en cuenta que no se determina en ella hasta dónde puede actuar el Presidente de la cooperativa, ${ }^{20}$ máxime si tenemos en cuenta que tiene en sus manos facultades tan importantes como la de representar a la cooperativa y la de suscribir contratos a nombre de ella. Tal y como podemos apreciar se trata de la persona que tiene el poder de obligar con sus actos a la cooperativa. En consecuencia, con el reconocimiento al Presidente de estas funciones pueden correrse riesgos importantes, uno de ellos puede ser la extralimitación en su actuar y consecuentemente causar un perjuicio no solo a la cooperativa sino también a los socios, pues ni siquiera se determina cuál es el límite en la actuación de este representante.

Quizás el reconocimiento del Presidente como una figura suprema, sea una consecuencia más de la extrapolación de los esquemas de dirección que existen en el sector estatal de nuestra economía.

\section{II.4.2. JUNTA DIRECTIVA}

Es la Junta Directiva un órgano colegiado de dirección de la cooperativa, subordinado a la Asamblea General, integrado por un Presidente, que es a su vez el Presidente de la Asamblea General, un se-

19 Vid. Artículo 37 Decreto 309/12.

20 En el Derecho de Sociedades el límite que recibe la representación como facultad de órgano de administración consiste en el objeto social. Consideramos que bien puede extrapolarse esta concepción al Derecho Cooperativo. 
cretario, y demás miembros cuya determinación corresponde a la Asamblea. A diferencia de la Asamblea General y del órgano de administración, la creación de la Junta Directiva dependerá de las características de la cooperativa, en particular se constituirá en aquellos casos en que la cooperativa posea más de sesenta socios.

Un aspecto interesante para el funcionamiento de todo órgano resultan sus atribuciones, las que generalmente se suelen determinar en las legislaciones, sin perjuicio de que en los estatutos puedan incorporarse otras. La asignación de facultades a cada órgano es un reflejo del principio de distribución de competencias que sin lugar a dudas pretende impedir la intromisión de un órgano en las funciones del otro. Ahora bien, ¿qué sucede con las facultades de la Junta Directiva? Pues bien, no se encuentran determinadas en la legislación que regula las cooperativas no agropecuarias en nuestro país, el legislador solo la reconoce como el órgano encargado de la dirección de la cooperativa. Sin embargo, al analizar la Asamblea General pudimos constatar que el legislador reconoce a dicho órgano como el órgano superior de dirección, podríamos preguntarnos entonces, ¿en una cooperativa integrada por más de 60 socios, qué papel juega la junta directiva, si coexiste con la Asamblea General? ¿Frente a la Asamblea General, será necesaria la constitución de la Junta Directiva? ¿Qué rol desempeñan la Asamblea y la Junta Directiva, si ambos son reconocidos legalmente como órganos de dirección de la cooperativa? Sólo nos queda claro de la lectura del artículo, su composición; la misma estará integrada por un presidente, un secretario y demás miembros que la Asamblea determine.

Otro elemento que refuerza nuestra hipótesis del Presidente como órgano unipersonal y omnipotente constituye el hecho que sí se regulen en la ley sus atribuciones como Presidente de la Junta Directiva, por ejemplo, se reconoce en el artículo 41 apartado c) como facultad del presidente la siguiente: «proponer o exigir que se adopten las medidas necesarias para dar cumplimiento a las recomendaciones que emanen de auditorías $u$ otras acciones de control externo que se realicen a la Cooperativa, y controlar su ejecución». Sin embargo, el legislador omite la regulación de las atribuciones de la Junta Directiva, mostrándose una vez más omiso y ambiguo, al no delimitar las atribuciones del órgano en cuestión. Tal indeterminación pudiera provocar una intromisión en su ámbito de actuación por la Asamblea General y del Presidente, pudiendo traer consigo una ineficiencia e inoperancia de la cooperativa. En el orden práctico, los cooperativistas cuyas cooperativas exceden de 60 miembros poseen poca claridad de las funciones que debe desempeñar la Junta Directiva. 


\section{II.4.3. Administrador o Consejo Administrativo}

Este órgano podrá adoptar la forma unipersonal o colegiada atendiendo a las características de la cooperativa. Será unipersonal en aquellas que no excedan de veinte miembros, en cambio aquellas integradas entre veinte y hasta sesenta socios podrán elegir un Consejo Administrativo. Tendrá a su cargo la gestión administrativa de la cooperativa. Además de las atribuciones que determine la ley, se le podrán reconocer otras en los estatutos.

La gestión, ${ }^{21}$ desde el punto de vista societario, ha sido definida por diversos autores, encontrando en ellas elementos comunes que, sin lugar a dudas, la tipifican. Una de las definiciones, que a nuestro juicio resulta completa, es la ofrecida por Rodríguez Artigas quien considera que «(...) cuando se habla de gestión o de administración de una sociedad mercantil se alude en términos muy amplios, al conjunto de actos, de variada índole, que se realizan sobre el patrimonio social y que tienden a la consecución del objeto social, ${ }^{22}$ por tanto, gestión y objeto social son términos que están estrechamente ligados y por ende los actos que comprenden la gestión no son aislados sino, que surgen del día a día de estos sujetos. Ahora bien, la gestión que regula el Decreto 309 a nuestro juicio es muy diferente de la que tiene a su cargo el órgano de administración de las sociedades capitalistas. En tanto, el órgano administrativo de la cooperativa no agropecuaria en Cuba será el titular de la gestión administrativa y por tanto se nos presenta como encargado de cuestiones de orden administrativas de la cooperativa. Suficiente es examinar las funciones que le son atribuidas a este órgano en el artículo $43.1^{23}$ del Decreto.

21 No debemos olvidar que, la gestión no es una categoría propia de las ciencias jurídicas, sino es un recurso de técnica empresarial, por lo que su extrapolación al campo del Derecho requiere de matices.

22 RODRÍGUEZ, J. L.: Consejeros Delegados, Comisiones Ejecutivas y Consejos de Administración, Editorial Montecorvo, p. 76, Madrid, 1971.

23 Artículo 43.1.- Es competencia del Consejo Administrativo o Administrador, la gestión administrativa de la Cooperativa y cualesquiera otras que le atribuyan los estatutos o los acuerdos de la Asamblea General y de la Junta Directiva cuando proceda. Tendrá como funciones específicas las siguientes: a) Elaborar los planes de producción o servicios, de ingresos y gastos de la Cooperativa y someterlos a la aprobación de la Asamblea General; b) informar periódicamente a la Asamblea General el estado de cumplimiento de los planes de producción de la Cooperativa; c) proponer a la Asamblea General la organización de las labores inherentes al objeto social de la Cooperativa, a cargo de los socios; d) advertir a la Asamblea General sobre las situaciones que perjudiquen el cumplimiento de los planes, así como la adquisición de insumos o la comercialización de los productos o servicios, u otras actividades que conforman el objeto social de la Cooperativa; e) informar a la Asamblea General los daños o pérdidas que ocurran, de bienes o derechos del patrimonio de la CooISSN: 1134 - 993X, Núm. 48/2014, Bilbao, págs. 227-243 
Por último, en lo que al órgano administrativo compete, consideramos oportuno analizar lo que dispone el artículo 43.2 del mencionado Decreto, el mismo plantea: «El Consejo Administrativo o Administrador, se subordina al Presidente o a la Junta Directiva cuando proceda.» En tal sentido consideramos que, la subordinación no debe ser, en ningún caso, al Presidente, en tanto no fue él quien eligió a este órgano, sino que ha sido fruto de la voluntad de los socios reunidos en Asamblea General. Somos del criterio que tampoco puede subordinarse a la Junta Directiva ya que este órgano no sustituye a la Asamblea General, sino que, además de la Asamblea, se constituye en aquellas cooperativas que poseen características propias, fundamentalmente atendiendo al número de socios y la complejidad de la actividad económica que desempeña. En todo caso, en ambos supuestos, la subordinación ha de ser a la propia Asamblea ya que es el órgano superior de dirección de la cooperativa y además el encargado de designar al administrador o los miembros del órgano administrativo.

\section{II.4.4. COMISIÓN DE CONTROL y FISCALIZACIÓN}

Este es un órgano, también necesario para el funcionamiento de la cooperativa. Podrá estar integrado por un socio o por varios de ellos, teniendo en cuenta el número de miembros que integren la cooperativa. Será designado por la Asamblea General. Tiene como función la supervisión del cumplimiento de las disposiciones legales, así como la utilización de los recursos financieros y materiales de la Cooperativa y rinde cuenta periódicamente de su actuar a la Asamblea. A ella no pueden pertenecer los miembros del resto de los órganos de dirección y administración. Se encuentra regulada en el artículo 45 del Decreto 309.

Consideramos que, sin lugar a dudas, el tema de los órganos de la cooperativa debe ser reformulado en una futura legislación sobre esta materia. Pues además de los comentarios que hemos realizado en los párrafos anteriores, somos del criterio que para la concepción de los mismos no se tuvo en cuenta las cooperativas integradas por reducido

perativa y sus causas, así como proponer las medidas dirigidas a obtener el resarcimiento correspondiente; f) adoptar medidas organizativas encaminadas a favorecer el debido cumplimiento de los acuerdos de la Asamblea General; g) contratar los trabajadores eventuales, según el marco regulatorio que se aprueben en los estatutos; h) controlar el cumplimiento de los deberes generales y demás tareas que se atribuyan a cada socio, así como, en su caso, de las labores de los trabajadores eventuales de la Cooperativa e in-formar de su resultado a la Asamblea General, al Presidente o a la Junta Directiva cuando proceda; i) rendir cuentas periódicamente a la Asamblea General sobre el resultado de su desempeño; y j) cualquier otra que resulte necesaria y se recoja en los estatutos de la Cooperativa. 
número de miembros. Partiendo del supuesto que todos los asociados deben integrar los órganos de la cooperativa y que, además, todos los órganos deben constituirse en ella; en una cooperativa que se cree con el límite mínimo de miembros, es decir tres, sucederá que todos integrarán los mismos órganos y esto puede traer consigo un entrelazamiento de funciones lo que podrá incidir en el funcionamiento de estos órganos así como también en lo relativo a la solución de conflictos.

Analizada a grandes rasgos la regulación de los órganos de la cooperativa contenida en la legislación, la autora considera que en una futura regulación jurídica debe ser un aspecto que requiere ser modificado, y tomando como base las experiencias que se logren acumular en este período, se dicte una normativa que regule este particular de manera más coherente.

\section{Ideas conclusivas}

Las cooperativas no agropecuarias, en nuestro sistema económico son de reciente aparición, por lo que las experiencias prácticas obtenidas de su funcionamiento no son del todo suficiente. No obstante, podemos afirmar que la legislación que la regula es, omisa y poco coherente, lo que puede incidir en su funcionamiento $y$, en consecuencia, distorsionar los hermosos contornos de la figura en cuestión. A partir de las experiencias que se logren acumular en este período de puesta en práctica de manera experimental de la figura, se publique una legislación general que de un modo coherente, armónico regule de manera unificada el fenómeno cooperativo, toda vez que hoy, en Cuba, coexisten varias normativas para un mismo fenómeno: el cooperativismo.

Apostar por el cooperativismo esta autora considera que es atinado, constituye una alternativa viable para lograr el desarrollo económico de nuestro país. Para ello, además de una legislación coherente, es necesario realizar acciones encaminadas a lograr una educación de la población en general acerca de lo que es el cooperativismo, así como a capacitar a las personas que de una u otra manera intervienen en el proceso de constitución y su funcionamiento. Estas son indispensables y de seguro permitirán un uso adecuado de la figura objeto de análisis. 


\section{Bibliografía}

AMORÍN, M.; ALGORTA, P.: Sociedades Cooperativas Sistema y Derecho Cooperativo, Ed. La Ley Montevideo, 2010, (versión de pre impresión facilitada por los autores en medio magnético para la presente investigación)

CRACOGNA D.: Régimen Jurídico de las Cooperativas, Buenos Aires, 1990

LLUIS, J.: Derecho de Cooperativas, Tomo I, Editorial Bosch, Barcelona 1972.

GADEA, E; SACRISTÁN; F.y VARGAS, VASSEROT, C.: Régimen jurídico de la sociedad cooperativa del siglo XX I. Realidad actual y propuestas de reforma, Ed. Dykison, Madrid, 2009.

PIÑERO, C.: Cooperativas y Socialismo, Una mirada desde Cuba, Editorial Caminos, La Habana, 2012

RODRÍGUEZ, J. L.: Consejeros Delegados, Comisiones Ejecutivas y Consejos de Administración, Editorial Montecorvo, Madrid, 1971.

RODRÍGUEZ, O.: La cooperativa como figura jurídica. Perspectivas constitucionales en Cuba para su aprovechamiento en otros sectores de la economía nacional diferentes al Agropecuario, Dykinson, S.L., Madrid, 2011 SánCHEZ Calero, F. y Sánchez-Calero, J.: Instituciones de Derecho Mercantil, Tomo I, Editorial Thomson-Aranzadi, Edición, Navarra, 2006.

\section{TEXTOS Legales}

«Ley de cooperativas de Argentina» No. 20337/73 de 2 de mayo de 1973.

«Ley de sociedades cooperativas de España» No. 27/99 de 16 de julio de 1999.

«Ley general de sociedades cooperativas de México» de 3 de agosto de 1994.

Decreto Ley 305 de 15 de noviembre de 2012, publicado en Gaceta Oficial Extraordinaria No. 053 de 11 de diciembre de 2012.

Decreto 309 de 28 de noviembre de 2012, publicado en Gaceta Oficial Extraordinaria No. 053 de 11 de diciembre de 2012. 